\section{AB080. Relationships between intravaginal ejaculatory latency time and National Institutes of Health-Chronic Prostatitis Symptom Index in the four types of premature ejaculation syndromes: a large observational study in China}

\author{
Jingjing Gao, Xiansheng Zhang \\ Department of Urology, The First Affiliated Hospital of Anhui \\ Medical University, Hefei 230000, China
}

Background: We assessed the associations between intravaginal ejaculatory latency time (IELT) and National Institutes of Health-Chronic Prostatitis Symptom Index (NIH-CPSI) in men with different premature ejaculation (PE) syndromes.

Methods: From September 2011 to September 2012, a total of 4,000 men were enrolled from the Anhui province of China. Subjects were required to complete a verbal questionnaire, including demographic information, medical and sexual history [e.g., IELT, and self-estimated scales (e.g., NIH-CPSI)].

Results: Of 3,016 of the men evaluated, $25.80 \%$ complained of PE. Distribution of the four PE syndromes among men with complaints of $\mathrm{PE}$ was as follows: lifelong premature ejaculation (LPE), 12.34\%; acquired PE (APE), $18.77 \%$; natural variable PE (NVPE), $44.09 \%$; prematurelike ejaculatory dysfunction (PLED), 24.81\%. Men with complaints of PE reported worse NIH-CPSI scores, and lower IELT than men without complaints of $\mathrm{PE}(\mathrm{P}<0.001$ for all). Moreover, total and subdomain scores of NIHCPSI were higher in men with APE, and IELT was higher in men with PLED. IELT was negatively associated with NIH-CPSI scores in men with complaints of PE. Negative relationships between total and subdomain scores of NIHCPSI, and IELT were stronger in men with APE (total scores: adjusted $\mathrm{r}=-0.68, \mathrm{P}<0.001$; pain symptoms: adjusted $\mathrm{r}=-0.70, \mathrm{P}<0.001$; urinary symptoms: adjusted $\mathrm{r}=-0.67$, $\mathrm{P}<0.001$; quality of life impact: adjusted $\mathrm{r}=-0.64, \mathrm{P}<0.001$ ).

Conclusions: Men with complaints of $\mathrm{PE}$ reported worse NIH-CPSI scores than men without complaints of PE. Relationships between IELT and NIH-CPSI scores were strongest in men with APE.

Keywords: Premature ejaculation (PE); intravaginal ejaculatory latency time (IELT); National Institutes of Health-Chronic Prostatitis Symptom Index (NIH-CPSI)

doi: 10.21037/tau.2018.AB080

Cite this abstract as: Gao J, Zhang X. Relationships between intravaginal ejaculatory latency time and National Institutes of Health-Chronic Prostatitis Symptom Index in the four types of premature ejaculation syndromes: a large observational study in China. Transl Androl Urol 2018;7(Suppl 5):AB080. doi: 10.21037/tau.2018.AB080 